\title{
A prediction model to identify people with spinal cord injury who are at high risk of dying within 5 years of discharge from hospital in Bangladesh
}

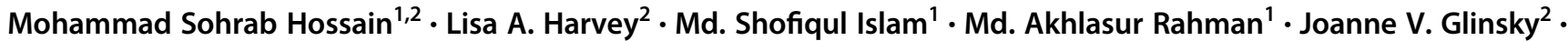 \\ Robert D. Herbert ${ }^{3}$
}

Received: 13 July 2018 / Revised: 3 October 2018 / Accepted: 8 October 2018 / Published online: 31 October 2018

(c) International Spinal Cord Society 2018

\begin{abstract}
Study design Mixed retrospective and prospective cohort study.

Objectives To determine 5-year survival after hospitalisation with spinal cord injury (SCI) in Bangladesh and to develop a prediction model to identify people at high risk of dying within 5 years.

Setting Bangladesh.

Methods Medical records were used to identify people with SCI admitted to a hospital in Bangladesh in 2011. Participants or their family members were contacted $>5$ years after discharge to determine vital status or date of death. Survival from time of discharge was estimated with Kaplan-Meier curves. A linear model of the log odds of death within 5 years of discharge was constructed and internally validated.

Results Of the 345 people who were admitted and survived to discharge in 2011, 342 (99\%) were accounted for 5 years later: $74(22 \%)$ had died (survival $=78 \%$; 95\% CI 74-82\%). Sixty nine of the 223 participants who were wheelchairdependent at discharge had died (survival $=69 \%$; 95\% CI 62-75\%). A parsimonious model predicted survival as a function of age and mode of mobility at discharge (wheelchair-dependent or ambulant). The odds of dying increased by a factor of 1.6 (95\% CI, 1.3-2.0) with every decade of age and by a factor of 12.6 (95\% CI, 4.8-32.9) if wheelchair-dependent. The model had good calibration and discrimination.

Conclusion The risk of dying after discharge from hospital with SCI in Bangladesh is high, especially among older, wheelchair-dependent people. A simple prediction model discriminates those at high risk of dying within 5 years.
\end{abstract}

\section{Introduction}

It is widely assumed that survival after spinal cord injury (SCI) in low-income and middle-income countries (LMICs) is poor [1, 2], but this assumption is based on sparse evidence. Two systematic reviews have examined survival following SCI [2, 3]. These reviews reported the results of 10 primary studies that provided estimates of 1-year

Lisa A. Harvey

1.harvey@usyd.edu.au

1 Centre for the Rehabilitation of the Paralysed, Savar, Bangladesh

2 John Walsh Centre for Rehabilitation Research, Sydney Medical School/Northern, University of Sydney, Sydney, NSW, Australia

3 Neuroscience Research Australia (NeuRA), Randwick, Sydney, NSW, Australia survival ranging from $79 \%$ to $100 \%$ and estimates of 5-year survival ranging from $85 \%$ to $96 \%$. Most of the primary studies included in these reviews were population-based studies conducted in high-income countries (HICs).

Few studies have estimated survival after SCI in LMICs. One retrospective study found that 5-year survival measured from time of injury of 490 patients with traumatic SCI referred to a rehabilitation centre in Southern India was $86 \%$ [4]. Another retrospective study of 422 patients with traumatic SCI admitted to one of the two hospitals in Nigeria found that 6-month survival measured from time of admission to hospital was $66 \%[5,6]$. However, both studies excluded patients who were not followed up after discharge, potentially biasing the estimates of survival. A study conducted in Brazil recently reported an $83 \%$ survival rate in a cohort of 343 patients discharged from hospital with a mean (SD) follow-up time of 4.8 (3.3) years [7]. The 
5-year survival rate was $82 \%$ (estimated from the Kaplan-Meier curve).

In 2014, we retrospectively identified all people $(n=$ 371) admitted in 2011 with a recent SCI to a hospital in Bangladesh that specialises in care of people with SCI. Of those discharged alive from hospital, $97 \%$ were followed up at least 2 years from discharge. Two-year survival, measured from the time of discharge, was $83 \%$ [8]. The present study reports 5-year survival data from the same cohort.

Prediction models that identify those people most likely to die soon after discharge could be used to inform life plans and to target provision of preventive interventions. Studies conducted in HICs show that age, gender and neurological loss are strong predictors of survival [3]. It is not known whether the same factors are strong predictors of survival in LMICs.

The aims of this study were to determine 5-year survival of people with SCI discharged from a specialised SCI hospital in Bangladesh and to develop a simple prediction model that could be used at discharge to identify those at high risk of death within 5 years.

\section{Methods}

The study was a mixed retrospective and prospective longitudinal cohort study [8]. Ethical permission was received from the Centre for Rehabilitation of the Paralysed Ethics Committee (approval number CRP-R\&E-0401-218) prior to the commencement of the study. Informed consent was received from living participants. If potential participants had died, consent was obtained from close family members. The study was conducted in accordance with the Declaration of Helsinki Principles.

The cohort has been previously described [8]. In brief, the cohort was drawn from patients admitted with an SCI to the Center for the Rehabilitation of the Paralysed (CRP), Bangladesh in 2011. Patients were excluded from the cohort if they sustained their injury $>1$ year prior to the date of admission or if they died prior to discharge. The CRP is the only specialised centre for people with SCI in Bangladesh. It admits approximately 390 patients with recent SCI each year. Most patients are admitted within a few days of injury but some are referred from other hospitals up to 6 months after injury. The initial cohort was retrospectively identified from three sources that were cross-checked. The first source was hospital admission records, the second was the social welfare department's records and the third was the detailed day-to-day medical files of admitted patients. The use of three sources increases the likelihood that we were able to identify all patients eligible to participate in the study.

The outcome of interest was survival 5 years after discharge from the CRP. Five-year survival was ascertained by telephoning participants between November 2017 and May 2018, at least 5 years after discharge. If a participant could not be contacted by telephone, immediate family members were telephoned to either obtain the contact information of the participant or to verify that the participant had died and to ascertain the date of death. When it was not possible to contact participants or their families by telephone, a home visit was conducted.

Five candidate predictors of survival were measured at discharge. We only considered as candidate predictors those variables that were routinely measured and recorded prior to discharge and that could in the future be easily collected at the time of discharge in other LMICs. Our choice of predictors was also guided by the literature and other studies from LMICs and HICs (for a summary of this literature, see Table 4 of ref. [3]) [2, 9, 10].

The candidate predictors were:

1. Type of lesion: classified as either paraplegia or tetraplegia. These data were obtained from the medical records.

2. Mobility at discharge: classified as either wheelchairdependent or ambulant. These data were obtained by asking participants at their 2-year follow-ups the following question (in Bangla): "On discharge, did you require a wheelchair for mobility on a daily basis?" For participants who had died, medical records were used to determine mobility on discharge (see previous publication for details [8]).

3. Gender: determined from the medical records but checked at the 2-year and 5-year follow-ups.

4. Age: determined from the medical records.

5. Cause of SCI: classified as either traumatic or nontraumatic. This was determined from the medical records.

The candidate predictors were treated as dichotomous variables, except age which was treated as a continuous variable. The American Spinal Injuries Association Impairment Scale was not used as a candidate predictor because we were concerned about relying on the medical records for these data and because this information could not be easily collected at the time of discharge, limiting the potential usefulness of predictions based on this variable.

\section{Data analysis}

Data were analysed using Stata v13.1. The cumulative probabilities of survival and hazard rates, stratified by mobility, were calculated using the Kaplan-Meier method.

To develop a prediction model, we ascertained who was alive 5 years after discharge. Univariate analyses were conducted to quantify the association between each 
predictor and survival at 5 years, expressed as an odds ratio. All predictors were then entered into a multivariate logistic model. (We used a logistic model, rather than a Cox model or a parametric survival model, because Cox models do not explicitly model outcomes and because parametric survival models are less easily converted into simple, clinically useful prediction rules.) The dependent variable was death within 5 years. Of the five candidate predictors, four (type of lesion, mobility at discharge, gender and cause of injury) were binary variables; the remaining predictor (age) was a continuous variable. For parsimony, we assumed that age had a linear effect. Bootstrap variable selection was used to select predictors. This involved applying a backwards stepwise selection procedure ( $p$ to remove $>0.2$ ) to models developed on each of 1000 bootstrap replicates of the original data set. The frequency with which each of the five predictors was selected across all bootstrap replicates was tabulated. Predictors that were retained in at least $80 \%$ of the bootstrap samples were included in the final model.

The calibration and discrimination of the model were examined to determine how well the model performed. Calibration reflects whether the predicted probabilities correspond with observed probabilities. It was assessed with Hosmer-Lemeshow test and the user-written calibrationbelt command in Stata [11, 12]. Discrimination, which reflects how well the model distinguishes between people who did and did not die within 5 years of discharge, was quantified with the area under the Receiver Operator Curve (AUC).

Naive estimates of discrimination were obtained by applying the prediction model to the original data set. Optimism-corrected estimates of discrimination were obtained by calculating, in each of the 1000 bootstrap replicates, the degree of optimism (i.e., the difference between the discrimination of the model applied to the bootstrap sample on which the model was developed and the discrimination of the model applied to the original sample) and then subtracting the mean optimism from the naive estimate [13].

\section{Results}

\section{Survival}

Three hundred and sixty eight people were admitted to CRP with recent SCI in 2011. Of these, 345 survived until discharge and constituted the inception cohort. The baseline characteristics of the cohort are shown in Table 1. Two hundred and twenty three participants were wheelchairdependent at the time of discharge. (See the legend of Table 1 for details about the small discrepancies between this paper and our previous publication [8].) All but three participants were accounted for at the 5-year follow-up. That is, 342/345 participants (99\%) contributed survival data until they died or were followed up. Median follow-up time was 6.1 years (interquartile range (IQR), 5.6-6.4 years) and total person-time of follow-up was 1815 years.

Seventy four participants died within 5 years of discharge. Five-year survival from time of discharge was $78 \%$ (95\% confidence interval (CI), 74-82\%). The mortality rate was 0.04 deaths per person-year $(95 \% \mathrm{CI}, 0.04-0.56$; Fig. 1). The 5-year survival for those who were wheelchairdependent was $69 \%$ (95\% CI, 62-75\%), whereas 5-year survival for those who were ambulant was $96 \%$ (95\% CI, 90-98\%; Fig. 1). The hazard of dying, determined from the whole cohort, was slightly higher in the first few months after discharge but was nearly constant thereafter.

Table 1 describes the common causes of death. The most common causes of death were sepsis secondary to pressure ulcers $(n=31 ; 42 \%)$ and respiratory-related problems $(n=$ $11 ; 15 \%)$.

\section{Prediction model}

The results of the univariate and multivariate analyses are shown in Table 2 . The variable selection procedure dropped gender, cause of SCI (traumatic or non-traumatic) and type of lesion (tetraplegia or paraplegia) from the model. The final multivariate model therefore included mobility at discharge and age. Both these variables were included in all bootstrap replicates. In univariate analyses, the odds of dying within 5 years among patients who were wheelchairdependent was 10.5 (4.1-26.9) times the odds of dying within 5 years among patients who were ambulant. The odds of dying within 5 years increased by a factor of 1.5 (1.2-1.8) with every decade of age. In the multivariate analyses, the corresponding odd ratios were $12.6(4.8-32.9)$ and $1.6(1.3-2.0)$. Fig. 2 shows the predicted probability of survival at 5 years as a function of age and mobility at discharge.

The prediction model differentiated quite well between participants who survived and who did not. Fig. 3 shows the distributions of the predicted probabilities of surviving for participants who did and did not survive to 5 years. The naive estimate of the AUC was 0.79 and the optimismadjusted estimate of the AUC was 0.78. The prediction model also appeared to be reasonably well calibrated. Hosmer-Lemeshow test was not statistically significant ( $p$ $=0.81$ ). Fig. 4 shows that the observed probability of dying within 5 years was similar to the predicted probability of dying within 5 years. This was true at all levels of predicted probability of dying. 
Table 1 Characteristics of participants included in the study

\begin{tabular}{|c|c|c|c|c|}
\hline & All participants ${ }^{\mathrm{a}}$ & $\begin{array}{l}\text { Alive at } 5 \\
\text { years }\end{array}$ & $\begin{array}{l}\text { Dead at } 5 \\
\text { years }\end{array}$ & $\begin{array}{l}\text { Lost to follow- } \\
\text { up }\end{array}$ \\
\hline Count, $n(\%)$ & 345 & 268 & 74 & 3 \\
\hline \multicolumn{5}{|l|}{ Gender, $n(\%)$} \\
\hline Female & $39(11 \%)$ & $31(12 \%)$ & $8(11 \%)$ & - \\
\hline Male & $306(89 \%)$ & $237(88 \%)$ & $66(89 \%)$ & $3(100 \%)$ \\
\hline \multicolumn{5}{|l|}{ Diagnosis, $n(\%)$} \\
\hline Paraplegia & $212(61 \%)$ & $171(64 \%)$ & $38(51 \%)$ & $3(100 \%)$ \\
\hline Tetraplegia & $133(39 \%)$ & $97(36 \%)$ & $36(49 \%)$ & - \\
\hline \multicolumn{5}{|l|}{ Cause of SCI } \\
\hline Traumatic & $327(95 \%)$ & $256(96 \%)$ & $69(93 \%)$ & $2(67 \%)$ \\
\hline Non-traumatic & $18(5 \%)$ & $12(4 \%)$ & $5(7 \%)$ & $1(33 \%)$ \\
\hline \multicolumn{5}{|l|}{ Mobility at discharge, $n(\%)$} \\
\hline Ambulant & $122(35 \%)$ & $116(43 \%)$ & $5(7 \%)$ & $1(33 \%)$ \\
\hline Wheelchair-dependent & $223(65 \%)$ & $152(57 \%)$ & $69(93 \%)$ & $2(67 \%)$ \\
\hline \multicolumn{5}{|l|}{ Mobility and diagnosis, $n(\%)$} \\
\hline Ambulant and paraplegia & $74(21 \%)$ & $68(25 \%)$ & $5(7 \%)$ & $1(33 \%)$ \\
\hline Ambulant and tetraplegia & $48(14 \%)$ & $48(18 \%)$ & - & - \\
\hline $\begin{array}{l}\text { Wheelchair-dependent and } \\
\text { paraplegia }\end{array}$ & $138(40 \%)$ & $103(39 \%)$ & $33(44 \%)$ & $2(67 \%)$ \\
\hline $\begin{array}{l}\text { Wheelchair-dependent and } \\
\text { tetraplegia }\end{array}$ & $85(25 \%)$ & $49(18 \%)$ & $36(49 \%)$ & - \\
\hline Age (years), median (IQR) ${ }^{\mathrm{d}}$ & $34(25-44)$ & $30(22-40)$ & $40(32-50)$ & $30(30-38)$ \\
\hline $\begin{array}{l}\text { Time from injury to admission } \\
\text { (days), median (IQR) }\end{array}$ & $12(4-35)$ & $12(3-35)$ & $12(5-37)$ & $33(1-68)$ \\
\hline $\begin{array}{l}\text { Length of hospital stay (months), } \\
\text { median (IQR) }\end{array}$ & $3.2(1.9-4.4)$ & $3.2(2.0-4.4)$ & $3.3(1.5-4.5)$ & $4.5(3.9-5.3)$ \\
\hline \multicolumn{5}{|l|}{ Cause of death, $n$} \\
\hline Pressure ulcer & - & - & 31 & - \\
\hline Respiratory related & - & - & 11 & - \\
\hline Other & - & - & 8 & - \\
\hline Unknown & - & - & 24 & - \\
\hline
\end{tabular}

${ }^{a}$ Our previous publication [8] involving the same cohort indicated that the number surviving to discharge was 350. The discrepancy of five people is because two people who we had been unable to find in our previous publication were found to have died prior to discharge. In addition, three people who were previously included were found to have not met the inclusion criteria (their injuries were $>1$ year prior to admission to CRP)

${ }^{\mathrm{b}}$ Includes seven people who were alive 5 years after their discharge dates but died by the time they were contacted for follow-up

${ }^{c}$ Includes one participant who is known to have died but whose date of death was not known

'Some participants' date of births were estimated because they did not know their date of birth

\section{Discussion}

This study provides estimates of survival 5 years after discharge from a specialised SCI hospital in Bangladesh. The estimates are based on a near-complete follow-up of an inception cohort. The data are valuable because of the scarcity of information from LMICs about survival after SCI $[2,3,7]$.

The data show that, among people discharged from CRP after a SCI, about 1 in 20 of those who are ambulant die within 5 years, whereas 1 in 3 of those who are wheelchairdependent die within 5 years. Overall, $78 \%$ of participants survived at least 5 years. These data might appear to be comparable with the results from HICs. For example, two recent reviews report 1-year and 5-year survival in people with SCI ranging from $79 \%$ to $100 \%$ [2, 3]. However, our estimates of survival are from time of discharge- they do not include the risk of dying prior to hospitalisation or in hospital (21 people or $5 \%$ of the original cohort died in hospital) [8]. The restriction of our cohort to those who 


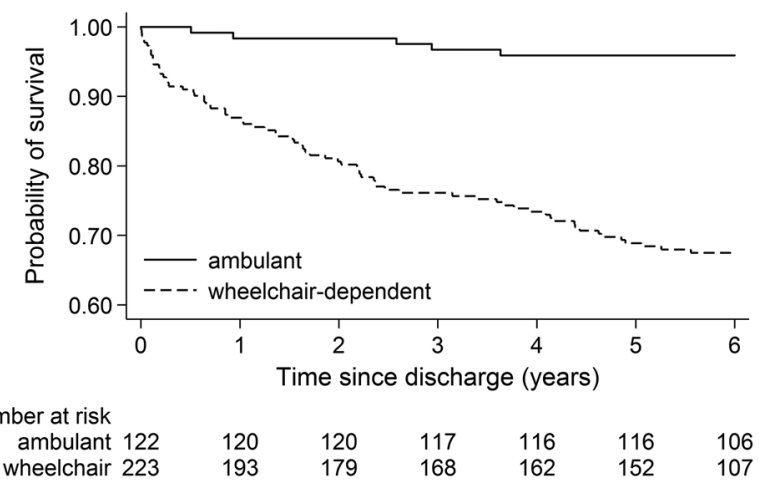

Fig. 1 Probability of survival stratified by mobility at discharge (i.e., ambulant or wheelchair-dependent)

survived until discharge probably explains why our cohort is younger (median age 34 years, IQR, 25-44 years) and less disabled than other cohorts. Older and more disabled people may not have survived long enough after injury to be admitted to CRP. While the estimates of survival from time of discharge provided by the current study are useful for post-discharge planning, estimates of survival from time of injury or time of hospitalisation would also be useful. Such studies are difficult to conduct in LMICs because they require well-coordinated health-care systems, integrated medical records and country-wide data registries.

The most common cause of death was sepsis due to pressure ulcers. It is widely recognised that pressure ulcers are a major problem after discharge from hospital with an SCI in LMICs [1, 14]. Participants in the current cohort were provided with good education and equipment for the prevention of pressure ulcers before discharge. Thus, while the proportion of deaths in the current cohort caused by pressure ulcers was high, it may be higher in other LMICs where good education and equipment for the prevention of pressure ulcers is not routinely provided. Nonetheless, there is an urgent need to reduce mortality rates due to pressure ulcers after discharge. To this end, we are currently conducting a clinical trial $(n=410)$ in Bangladesh to determine whether 2-year mortality can be reduced following discharge by providing patients with ongoing communitybased support following discharge $[15,16]$. The trial will be completed in early 2020 .

We identified a parsimonious model that predicted 5-year survival from just two variables: age and mobility status at discharge. This is consistent with the findings of other studies that have identified age and extent of neurological loss as the strongest predictors of survival [3]. We used mobility status as a crude measure of neurological loss because we had access to data on mobility status and because we wanted a prediction model that could be used without having to conduct a full neurological assessment at discharge. It is easier to determine mobility status on discharge than to conduct a full neurological assessment according to the International Standards for the Neurological Classification of Spinal Cord Injuries. The simplicity of the model is its strength. Health-care professionals could easily use Fig. 2 to predict a patient's probability of survival 5 years after discharge based on age and mobility status on discharge. Patients at high risk of dying might be monitored more closely and provided with additional health care. This is important for LMICs that do not have the resources to provide highly specialised and intensive follow-up care for all discharged patients. There is a suggestion that this type of prioritised follow-up is already happening in countries like Afghanistan [17].

It was interesting that type of SCI (tetraplegia or paraplegia) was not retained in the final prediction model even though type of SCI reflects neurological loss. More than half of those with tetraplegia in our cohort were able to walk and most of the people with tetraplegia who were unable to walk had low cervical injuries. This is probably because, in Bangladesh, people with high cervical lesions typically do not survive more than a few days after injury and are not commonly admitted to CRP. For this reason, there may not have been a large difference in the neurological status of people with tetraplegia and paraplegia in our cohort. This explanation was provided by others [3] who observed similar findings [18]. We did not find that gender or cause of SCI were strong predictors of mortality, even though a recent review found that mortality rates were consistently higher in men than in women and in those who sustained a non-traumatic SCI compared to a traumatic SCI [2]. This may reflect the small number of women (11\%) and people with non-traumatic SCIs (5\%) in our cohort. Nonetheless, our prediction model is broadly consistent with the findings of other studies that have predicted mortality from age at injury, neurological level and completeness of injury [3].

The model appears to be have an acceptable level of discrimination. The AUC of 0.78 implies that, in $78 \%$ of randomly selected pairs of study participants with discordant outcomes (pairs in which one person died within 5 years and the other did not), the person who died had the higher predicted probability of dying [19]. As a comparison, the widely used Framingham cardiovascular risk score has an AUC of 0.72-0.76 when used to predict cardiovascular events in New Zealand [20]. Estimates of discrimination were internally validated and adjusted for optimism with bootstrapping, making them more likely to apply to other samples from a similar population. Nonetheless, the model now needs to be externally validated by assessing the predictive performance of the model on samples drawn from other settings (e.g. rural hospitals) in other LMICs.

There are limitations to this study. Most importantly, the study was conducted at a single centre. Also, the cohort was identified retrospectively and some of the predictors were 
Table 2 Univariate and multivariate associations between candidate predictors and survival with 95\% CI

\begin{tabular}{|c|c|c|c|c|}
\hline \multirow[t]{2}{*}{ Candidate predictors } & \multirow{2}{*}{$\begin{array}{l}\text { Univariate analysis } \\
\text { Odds ratio }\end{array}$} & \multirow{2}{*}{$\begin{array}{l}\text { Percentage of bootstrapped } \\
\text { samples that retained predictor }\end{array}$} & \multicolumn{2}{|c|}{ Multivariate analysis } \\
\hline & & & Odds ratio & Regression coefficients \\
\hline Age (in 10-year increments) & $1.5(1.2$ to 1.8$)$ & $100 \%$ & $1.6(1.3$ to 2.0$)$ & $0.47(0.27$ to 0.68$)$ \\
\hline Mobility at discharge & $10.5(4.1$ to 26.9$)$ & $100 \%$ & $12.6(4.8$ to 32.9$)$ & 2.53 (1.57 to 3.49$)$ \\
\hline Type of lesion & 1.7 (1.0 to 2.8$)$ & $71 \%$ & - & - \\
\hline Gender & $1.1(0.5$ to 2.5$)$ & $30 \%$ & - & - \\
\hline Cause of injury & $1.5(0.5$ to 4.5$)$ & $52 \%$ & - & - \\
\hline Intercept & - & - & - & $-5.31(-6.67$ to -3.96$)$ \\
\hline
\end{tabular}

Also shown are the percentage of bootstrapped samples that retained each candidate predictor. The multivariate model was a logistic model. The dependent variable was death within 5 years of discharge. The reference groups for the binary mobility, diagnosis, gender and cause of injury variables are, respectively, ambulant, paraplegia, female and traumatic. The effect of age on log odds of death was assumed to be linear. Confidence intervals do not take account of model selection procedures

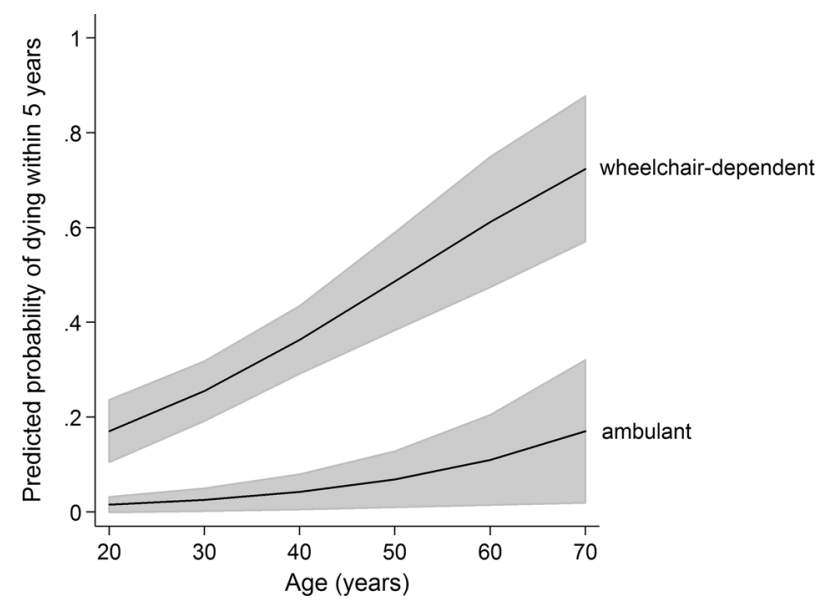

Fig. 2 Predicted probability of dying within 5 years of discharge based on age and mobility (ambulant or wheelchair-dependent) at discharge. The shaded areas are $95 \%$ CIs

collected retrospectively from medical records. As a consequence, it is possible that a small number of potentially eligible participants were not included in the cohort and that predictor data were inaccurate. In addition, we relied on family members to recall the date and cause of death because Bangladesh does not have a death registry. These limitations may have introduced small errors into the estimates of survival.

In conclusion, this study provides some of the first robust data on survival following discharge with an SCI in an LMIC. Approximately one in three patients who were wheelchair-dependent on discharge died within 5 years. We emphasise that this estimate is of survival from the time of discharge; it should not be used to predict survival from the time of injury or time of hospitalisation. A simple prediction model based on age and mobility at time of discharge coarsely discriminates between those who will and will not
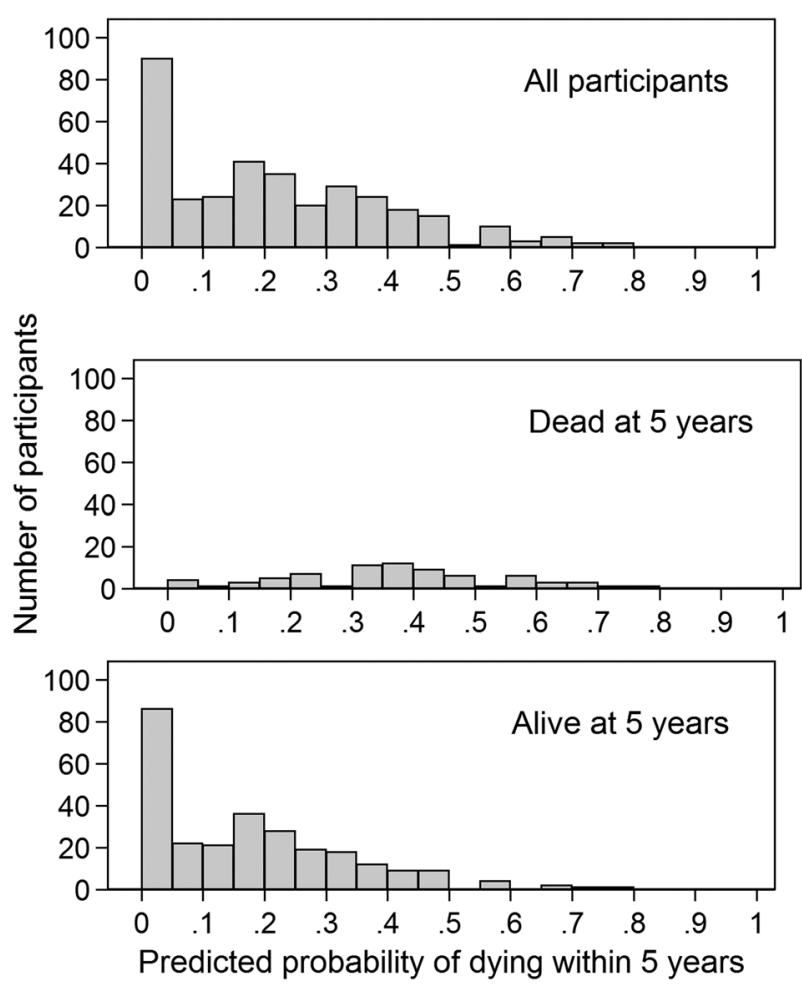

Fig. 3 Discrimination. The three histograms show the number of participants ( $y$ axis) as a function of the model-predicted probability of dying within 5 years ( $x$ axis) for all participants (top figure), those who died within 5 years (middle figure) or those who were alive at 5 years (bottom figure). If the prediction model was not discriminative, the distributions in the bottom two figures would be the same. If the model was perfectly discriminative, there would be no overlap of the two distributions. These graphs show that the model was moderately discriminative: the AUC was 0.78 , meaning that in $78 \%$ of all pairs of participants (one from each distribution) the person who died had a higher predicted probability of dying than the person who did not die

survive for 5 years. Individuals who are at high risk of dying within 5 years might be targeted for ongoing followup and care. 


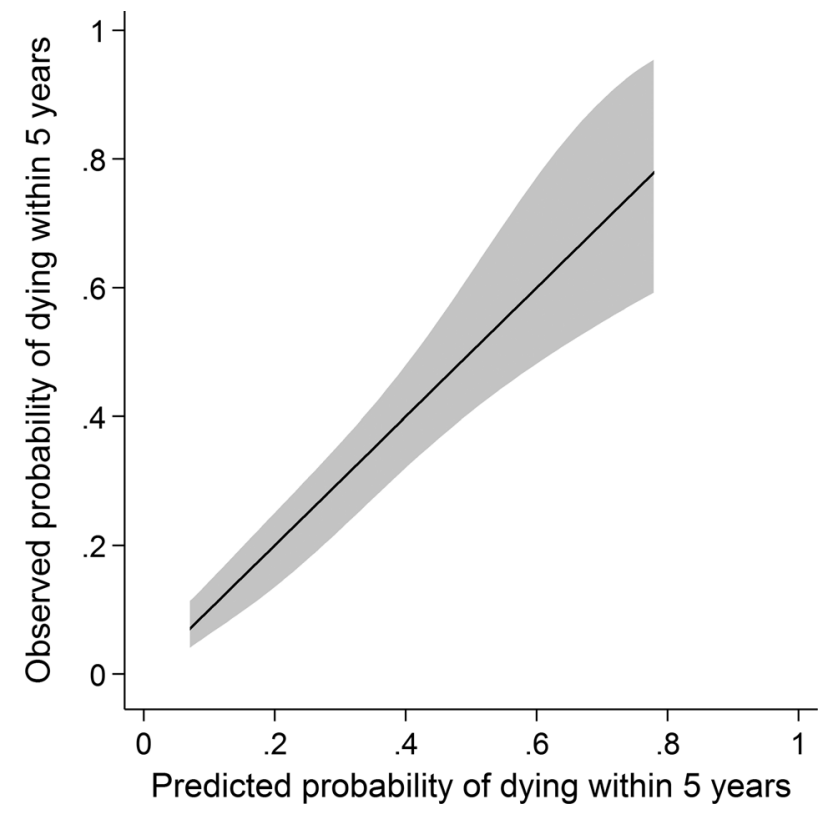

Fig. 4 Calibration of the model. Perfect calibration is represented by the diagonal line, because this is the line on which the predicted probability equals the observed probability (the line of identity). The shaded area is a calibration belt: it reflects the statistical uncertainty about the true relationship between the predicted probability of dying within 5 years and the observed probability of dying within 5 years [21]. The calibration belt includes the line of identity along its full length, meaning that there is no evidence of model miscalibration. The calibration belt only extends from predicted probabilities of $0.01-0.81$ because this is the range of participants' predicted probabilities of dying within 5 years

Data archiving The authors will consider all reasonable requests for data sharing.

Acknowledgements We thank the staff and patients from the Centre for the Rehabilitation of the Paralysed who were involved in this study. We also thank the staff of the Community Based Rehabilitation/ Social Welfare departments and the Medical Admission Unit for their diligent record keeping.

Author contributions MSH conceived the research question, designed the study, collected the data, analysed the data, interpreted the data and wrote the manuscript. LAH and RDH conceived the research question, designed the study, analysed the data, interpreted the data and wrote the manuscript. MSI and MAR collected the data and contributed to the research question, the design of the study, the interpretation of the data and the write-up of the manuscript. JVG contributed to the research question, the interpretation of the data and the write-up of the manuscript.

Funding This work was supported by Bridging Support Grants from The University of Sydney [171654 and 2013-00033].

\section{Compliance with ethical standards}

Conflict of interest The authors declare that they have no conflict of interest.
Statement of ethics Ethical permission was received from the Centre for the Rehabilitation of the Paralysed Ethics Committee (approval number CRP-R\&E-0401-218) prior to the commencement of the study. We certify that all applicable institutional and governmental regulations concerning the ethical use of human volunteers/animals were followed during the course of this research.

\section{References}

1. World Health Organization. International perspectives on spinal cord injury. Geneva: World Health Organisation; 2013.

2. Chamberlain JD, Meier S, Mader L, von Groote PM, Brinkhof MW. Mortality and longevity after a spinal cord injury: systematic review and meta-analysis. Neuroepidemiology. 2015;44:182-98.

3. van den Berg ME, Castellote JM, de Pedro-Cuesta J, MahilloFernandez I. Survival after spinal cord injury: a systematic review. J Neurotrauma. 2010;27:1517-28.

4. Barman A, Shanmugasundaram D, Bhide R, Viswanathan A, Magimairaj HP, Nagarajan G, et al. Survival in persons with traumatic spinal cord injury receiving structured follow-up in South India. Arch Phys Med Rehabil. 2014;95:642-8.

5. Nwadinigwe CU, Iloabuchi TC, Nwabude IA. Traumatic spinal cord injuries (SCI): a study of 104 cases. Niger J Med. 2004; 13:161-5.

6. Kawu A, Alimi M, Gbadegesin S, Salami O, Olawepo A, Adebule G. Risk Factors Predicting Mortality in Spinal Cord Injury in Nigeria. WebmedCentral ORTHOPAEDICS 2010;1(9): WMC00807 https://doi.org/10.9754/journal.wmc.2010.00807.

7. Leite V, de Souza D, Imamura1 M, Battistella L. Post-discharge mortality in patients with traumatic spinal cord injury in a Brazilian hospital - a retrospective cohort. Spinal Cord. 2018. https:// doi.org/10.1038/s41393-018-0183-y.

8. Hossain M, Rahman M, Herbert R, Quadir M, Bowden J, Harvey L. Two-year survival following discharge from hospital after spinal cord injury in Bangladesh. Spinal Cord. 2016;54:132-6.

9. Catz A, Thaleisnik M, Fishel B, Ronen J, Spasser R, Fredman B, et al. Survival following spinal cord injury in Israel. Spinal Cord. 2002;40:595-8.

10. O’Connor PJ. Survival after spinal cord injury in Australia. Arch Phys Med Rehabil. 2005;86:37-47.

11. Royston P, Moons KG, Altman DG, Vergouwe Y. Prognosis and prognostic research: developing a prognostic model. BMJ. 2009;338:b604.

12. Nattino G, Lemeshow S, Phillips G, Finazzi S, Bertolini G. Assessing the calibration of dichotomous outcome models with the calibration belt. Stata J. 2017;17:1003-14.

13. Steyerberg E. Clinical prediction models: a practical approach to development, validation, and updating. New York, NY: Springer; 2009.

14. Zakrasek EC, Creasey G, Crew JD. Pressure ulcers in people with spinal cord injury in developing nations. Spinal Cord. 2015; 53:7-13.

15. Hossain MS, Harvey LA, Liu H, Islam MS, Rahman MA, Muldoon S, et al. Protocol for process evaluation of CIVIC randomised controlled trial: Community based InterVentions to prevent serIous Complications following spinal cord injury in Bangladesh. BMJ Open. 2018;8:e24226.

16. Hossain MS, Harvey LA, Rahman MA, Muldoon S, Bowden JL, Islam MS. et al. Community-based InterVentions to prevent serIous Complications (CIVIC) following spinal cord injury in Bangladesh: protocol of a randomised controlled trial. BMJ Open 2016; 6: e010350. doi:010310.011136/bmjopen-012015010350. 
17. Michael M, Roth K. Against all odds: a qualitative study of rehabilitation of persons with spinal cord injury in Afghanistan. Spinal Cord. 2012;50:864-8.

18. Imai K, Kadowaki T, Aizawa Y. Standardized indices of mortality among persons with spinal cord injury: accelerated aging process. Ind Health. 2004;42:213-8.

19. Harrell FE Jr, Lee KL, Mark DB. Multivariable prognostic models: issues in developing models, evaluating assumptions and adequacy, and measuring and reducing errors. Stat Med. 1996;15:361-87.
20. Rabanal KS, Meyer HE, Pylypchuk R, Mehta S, Selmer RM, Jackson RT. Performance of a Framingham cardiovascular risk model among Indians and Europeans in New Zealand and the role of body mass index and social deprivation. Open Heart. 2018;5: e000821.

21. Nattino G, Finazzi S, Bertolini G. A new test and graphical tool to assess the goodness of fit of logistic regression models. Stat Med. 2016;35:709-20. 